\title{
1. Preparing for high impact change: the critical role of experiential learning and practice
}

\author{
Anthony F. Buono, Susan M. Adams, and \\ Gavin M. Schwarz
}

This book is intended to help prepare change leaders - at all organizational levels - to effectively deal with the myriad challenges inherent in the process of organizational change. While it has literally become a well-worn cliché that organizations and their management face unrelenting demands for change (Kerber and Buono, 2018), the simple reality - which is not so simple in terms of its impact on organizational life - is that the majority of change efforts fall well short of their intended goals. Despite a literal avalanche of research and managerial attention devoted to conceptualizing and empirically testing an array of change management practices (see Abrahamson, 2000; de Caluwé and Vermaak, 2002; Jamieson et al., 2016; King and Wright, 2007; Kotter and Cohen, 2002), successful organizational change often remains an elusive quest. Unfortunately, many of our educational efforts to develop the capabilities of students and early career executives to successfully deal with the subtleties, nuances, and complexities of organizational change similarly tend to fall well short of the need. Thus, in a "back to the future" spirit, this volume seeks to confront this challenge by resurrecting a powerful hands-on, immersive pedagogy: high impact change-related simulations and experiential exercises.

Effective organizational change involves a combination of understanding, learning and unlearning, and practiced behavior as part of the underlying conceptualization, formulation, and implementation processes. The book presents a series of exercises - each with background context, explicit directions, facilitator suggestions, and debriefing guidelines - that promote learning and developing readiness for change, from preparing people for change, understanding and managing resistance, grappling with cultural confines and confusion, dealing with communication challenges, and coping with change-related obstacles, to seeking buy-in for the change. Emphasis throughout the book is placed on developing change-related competencies. 
Some background for the volume is in order. It has long been recognized that change is an intrinsic factor in the long-term success and survival of organizations, leading to extensive frameworks and ways to deal with and manage organizational change, from such allegorical tales as Johnson's (1988) Who Moved My Cheese? and Kotter and Rathgeber's (2016) Our Iceberg is Melting, to conceptually oriented frameworks (e.g., Jamieson and Armstrong, 2010; Kerber and Buono, 2005, 2018; Kotter, 1996), to more empirically based approaches (see Beer, 2006; de Caluwé and Vermaak, 2002). The accepted view is that for the business world as well as society as a whole, the magnitude, speed, impact, unpredictability, and consequences of change are greater than ever before. Consequently, organizations face a whirlwind of changes in trying to keep their balance between the need to perform in the present amid the pressures of change moving forward. Yet, as noted above, evidence suggests that most organizational change efforts fall well short of intended targets, more often than not failing in some form.

On an individual level, despite this lack of success, we all experience continuous and repeated change in the workplace, which has led to the development of the field of change management: a systematic approach aimed at shaping the thinking of students, practitioners, and educators on the process of organizational change. This training and focus, however, has created a conundrum: when it comes to training people for organizational change, most attention is given to examining how to manage and lead change, often focusing on the impact of change. Based on the evidence of its failure, however, it seems that we still do a poor job at preparing people for the realities of change and the process of change itself. An underlying problem is that the themes and approaches in discussing "how to" change have remained constant for the past several decades. Plus ca change, plus c'est la meme chose - the more things change, the more things remain the same? Yet, at the same time, a rich tradition in preparing people for change - embedding them in exercises and experiences that simulate various aspects of the change experience - has seemingly faded from view, despite it being an increasingly necessary part of how we transfer knowledge.

With its focus on facilitating change, this book takes a step back in deconstructing and presenting a series of hands-on approaches to engaging change. On a personal level, if you have ever wondered how you can engage better with organizational change, whether you would like to instill a healthier response to the next change you face, or if you want to better understand how change can be a positive vehicle for organizations, then this book is perfect for you. Educators will find it useful in helping to create an active learning environment, directly engaging participants in the nuances and subtleties inherent in change processes. Grounded in experiential 
learning, it presents a forum for a broad array of stakeholders - educators, researchers, consultants, and practitioners - showcasing high impact methods for engaging with organizational change in its many contexts.

This book is the culmination of a series of successful Professional Development Workshops (PDWs) during the Academy of Management annual meeting for more than a decade. Beginning in 2005 in Honolulu, we honestly did not know what to expect when we ran the first PDW "That Was Great! High Impact Activities, Exercises and Approaches for Teaching or Consulting on Organizational Change" - co-sponsored by the Organization Development and Change Division and the Management Consulting Division. At an 8:00-10:00 am session on a Friday morning, with the lure of Diamond Head and Waikiki Beach dangling in front of Academy members, well over 100 people showed up to an overfilled room. Participants experienced five abbreviated exercises or simulations accompanied by interaction, facilitator suggestions, questions, and discussion. The session was energetic, with a noted buzz throughout the room and more than a few "Aha!" moments punctuated with laughter, openness, and sharing among colleagues. We clearly hit a need. We have run the PDW every year since then with similar sized audiences and reactions, at this point culminating in Chicago in 2018 - and our decision to co-write and edit this volume.

The objective of the book is to provide educators, consultants, and practitioners as well as students with a set of coherent and rigorous perspectives to better understand organizational change and changerelated processes through high impact exercises. At its foundation, while we acknowledge that there is a history of practitioners, educators, and scholars (especially those working in organizational development) engaging in techniques and exercises to help raise self-awareness of learning (see, e.g., Pfeiffer and Jones, 1972), this type of publication - a collection of techniques for experiential learning - is far less relevant today. Yet, paradoxically, our research suggests that people experiencing change both seek it out and avoid it, a dynamic that is often lost in academic and practitioner approaches. Most academic attention focuses on understanding the nature, content, and processes of change, often through applied cases; in essence, better understanding what change and its make-up look like, and how it was handled in a particular instance. In contrast, most practitioner interest has resulted in an emphasis on "toolkits" for managing change and change-making tactics from client experience. This book seeks to engage these two foci, personalizing the change experience through role play, simulation, and experiential exercises.

The overarching theme is learning from the change experience, focusing on the challenges involved in implementing and facilitating the change 
process: learning (about change) by doing. After establishing the basis of learning from change, the set of exercises presented and deconstructed encompass the fundamental features of organizational development and change in facilitating the improvement of an organization's problemsolving and renewal processes, through more effective and collaborative management of change. The range of exercises provides facilitators options for reaching those with learning styles which are often ignored. This collection raises the odds for giving everyone a chance to learn their own way (Boyatzis and Kolb, 1995) and to have a more lasting recall of the lessons.

A quote that has been attributed to Confucius - "I hear and I forget, I see and I remember, I do and I understand" - is still very relevant today, as individuals typically prefer different modalities for learning. As an example, Barbe et al. (1979) suggest that there are preferences to learn visually, auditorily, or kinesthetically - in other words, from seeing, listening, or doing. The choice, of course, need not present an either/or challenge. Kolb's (2014) model of the learning process, for example, envisions a cycle of learning that begins with concrete experience, followed by reflective observation, abstract conceptualization, and more active experimentation. This book offers exercises that capture the "doing" dimension of the learning cycle, supporting a hands-on approach to experiencing various aspects of the change experience in "safe" environments. The volume's emphasis on the importance of debriefing these exercises captures Kolb's notions of reflective observation and abstract conceptualization; examining and processing one's own experience and the experience of other participants. Within this context, it is important to remember that learning to understand and lead change from doing it in the classroom (or in training simulations) is much safer than in the organizational world where the impact of failure is much higher.

\section{THE VOLUME}

The book is divided into four parts that explore various ways to enhance our understanding of change through experiential learning, from initial thoughts about getting started in the process, to self-learning and personal development, and the challenges related to communicating change, to exploration of the human side of the change experience on multiple levels. The final part provides explicit direction for experiential learning's "end game" - a critical debriefing of the experience and exploration of lessons learned. 


\section{Experiential Learning as a Pedagogical Tool: Getting Started}

The first group of exercises, in Part I, has three chapters that set the stage for experiential learning. Following this introduction, Chapter 2 offers a historical perspective on the field of organization development (OD) that orients the rationale for this volume, focusing on the competencies of successful scholarpractitioner change agents. Ram Tenkasi, George Hay, and Eric Sanders describe how the pursuit of high impact change is a familiar quest within the field of organization development and change (ODC). Expectations that the results of any change initiative must be impactful are quintessential in today's organizations faced with pressure to demonstrate their financial value in hyper-competitive, global markets. Tenkasi and his colleagues assert that high levels of change impact can be realized through theoretically informed practice, implicitly drawing upon a key founding principle of ODC, "there is nothing as practical as a good theory" (Lewin, 1945, p. 126).

In contemporary times this basic Lewinian idea is expressed in the notion of actionable scientific knowledge. This concept refers to processes of knowledge creation that fulfill the criteria of the scientific community and also impact the business needs of the organization. Actionable scientific knowledge outcomes are both theoretical and practical, serving theoretical advancement while simultaneously impacting business needs of organizations. While there are multiple agents of actionable scientific knowledge, the chapter's focus is on ODC scholar-practitioners who are concerned with organizational change, applying theory to organizational change practice to create successful impact for both theory and practice. These professionals approach ODC from an integrated role as theoreticians, researchers, and change agents who use theory to influence practice. The chapter draws on two studies that reveal the competencies of these successful scholar-practitioners.

In Chapter 3, volume co-editor Anthony Buono looks at the use of icebreaker exercises as a way of initially engaging participants, opening them up and setting the stage for learning, reflection, and sharing. As he suggests, an underlying problem is that many, if not most, exercises of this nature typically make people feel ill at ease: instead of engaging them, they make participants feel awkward and uncomfortable. It is not that such exercises do not work or should not be used - as he suggests, "they can and they should" - but they need to be clearly related to and focused on the topic under study. As an example, the chapter presents a word association exercise - the Reaction-to-Change (RTC) inventory - that sets the stage for examining people's reactions to change, on both a general level as well as in relation to a specific change, and serves as a foundation for the type of learning upon which the volume is focused. 
Concluding this part of the book, Robert Marshak examines the power of experiential learning in enhancing theory-oriented courses. As he suggests, instructors in such courses are often challenged to communicate abstract concepts in ways that generate interest and enhance internalization, getting participants to focus on application rather than concept memorization. Yet, despite advances in learning methodologies, he argues that the read-lecture-discuss method still tends to be the preferred vehicle for conceptual learning. Unfortunately, the result is often people who can recite theories chapter and verse, but have difficulty finding any use for this knowledge beyond demonstrating that they are "educated." His chapter begins with a discussion of the importance of theory as a practical tool for engaging the world around us. Noting that when experiential methods are used, they are typically placed in sequential combinations with cognitive readings and discussions, Marshak proposes an alternative approach, one that seeks to simultaneously combine experiential and cognitive learning about theory, illustrating this approach with various design examples and offering suggestions for debriefing a simultaneous cognitive and experiential learning design.

\section{Self-Learning and Beyond through Change Exercises}

Part II includes four chapters that focus on specific experiential exercises with an emphasis on self-learning about the change process. Each of the chapters introduces and explains a seminal change theme, highlighting the change, its different mechanisms, and the value of different approaches to dealing with such change. The chapters detail the theme and potential for high impact change, explaining its basis and theoretical framework. The contributors deconstruct their high impact exercises, providing the reader with the basis for the exercise and its history (for example, classic exercise, a derivation from core theory), with a handson explanation of the exercise and method for debriefing. The chapters conclude with reflection on the underlying insights into our learning about the change.

Beginning with Chapter 5, Philip Mirvis focuses on enhancing selfawareness through storytelling and mask-making. The exercise involves participants creating personal autobiographies, telling stories about themselves, and creating masks that reflect how they see themselves, cultivating introspection and reflection. Drawing on specific instances where he used this approach, Mirvis illustrates how these exercises can engage emotions as well as cognitive understanding for the participants as well as others in their surroundings. As he suggests, enhancing self-awareness can also serve as a bridge to change; both self and organizational. Drawing on his 
experience, he notes that it is common for people to follow up such selfreflection with specific action plans, from self-improvement initiatives to ways to adapt to new organizational requirements.

Chapter 6 by volume co-editor Susan Adams shares a card game that involves multiple rounds of simulated organizational changes, with participants physically moving to different groups based on "winner" and "loser" status. The game gives participants opportunities to observe their own behaviors and emotions during change and to experience how others react; both to each other, and perceived norm violations. The debriefing discussion links these observations to change-related triggers as well as potential management techniques to counteract such tendencies. As she notes, by focusing on our reactions to change, the exercise increases awareness on multiple levels, from forming a better understanding of our own personal reaction to change and the different ways in which individuals react to change, to group influences on reactions to change and our own reactions to others' change-related reactions.

In Chapter 7, Matt Minahan outlines two related activities - the Argument of Obviousness and Dealing in Decades - that are designed to assist organizations in differentiating and integrating various parts of the system involved in an organizational change. As he argues, a significant need in any change effort, especially in terms of large-scale change, is to align the beliefs and perceptions of the myriad stakeholders involved to better enable the organization to take concerted action going forward. The underlying challenge, he points out, is facilitating conversations that help build a common understanding among people who may not know each other and may even feel threatened by those others, as well as building the necessary trust to allow such conversations to take place. The exercises in the chapter are intended to assist organizational members in establishing that trust, realizing and capitalizing on system complexity and the two underlying, seemingly contradictory, dimensions of organizational life: differentiation and integration.

The concluding chapter in Part II, Chapter 8 by Michael Manning and Melissa Norcross - the Mads, Glads, and Sads exercise - was originally designed as part of a large-scale change initiative, focusing on getting the "whole system in the room" to engage in system-wide action planning. The exercise creates the context for groups of any size to reflect on their personal organizing experiences - both emotionally as well as cognitively - with the intention of enhancing participant engagement and commitment in the change process. The exercise has an explicit focus on the central role of emotions in terms of dealing with, managing, and activating change. 


\section{Communicating Change}

The two chapters in Part III share exercises that focus on challenges related to communicating about and within change. In Chapter 9, Richard Dunford's Symbols exercise illustrates several of these challenges, from recognizing the importance of clearly communicating the objective of the change, to understanding the individual and organizational effects of miscommunication, including misunderstanding (management's objectives), frustration (even anger), demotivation, and underperformance against objectives, to understanding how inadequate communication can lead to the (mis)attribution of meaning to specific activities by organizational members. In the exercise, groups will only be able to complete the task by deciphering codes in their "rules," emphasizing the need to pay attention to how others communicate, understand how information ebbs and flows in an organization, and to take the time to understand the associated meanings of comments and the behavior of others.

Cynthia Martinez's Ball Game exercise in Chapter 10 presents an exercise using tennis balls to help participants experience the chaos of change, consider the different variables and/or circumstances affecting the change process, and the challenge of creating strategies and actions for changerelated planning, implementation, and evaluation. The Ball Game introduces critical dimensions of change, getting participants to experience what it feels like to encounter change and work through the important change-related communication functions, methods, and processes.

\section{The Human Side of Change: Strategy, Culture, and Change Recipients}

The five chapters in Part IV focus on the interpersonal side of change, offering exercises related to resource dependency, negotiating, conflict management, collaboration and the influence of individuals, culture and social networks.

In Chapter 11, Mary Nash, Michael Manning, and John Heiser apply Kurt Lewin's Force Field Theory to facilitate SWOT (strengths, weaknesses, opportunities, threats) analysis. Their methodology promises to be beneficial for a range of users, from leaders and managers, to organizational members and other stakeholders involved in the strategic planning process, as well as facilitators (internal or external) of such initiatives. Their approach also provides a useful road map - with possible routes and barriers - in planning for change, which can readily inform preparations for change and provide a starting point that can be revisited during change implementation.

Chapter 12 by Ann Feyerherm presents an exercise that faces participants with the opportunity and challenge of physically dealing with 
diminishing resources, in the form of paper squares on the floor that are taken away during the exercise. The task is to find creative solutions to problems precipitated by the removal of the squares. While simple (and somewhat reminiscent of the children's game of Musical Chairs), the exercise simulates reactions to typical resource decline, serving as a potential springboard to innovatively dealing with change. As Feyerherm emphasizes, the experience brings the phrase "necessity is the mother of invention" to life in a very real way.

In Chapter 13, David Jamieson, Jackie Milbrandt, and Nicole Zwieg Daly describe an exercise called the Culture and Change Challenge. The experiential activity is intended to help participants develop the awareness and skills needed to anticipate, observe, and leverage culture in organizational change. As participants explore the dynamics of culture and change through the exercise - described as an accelerated learning experience with differences, disorientation, and disconfirmation - they build greater cultural awareness, thinking, and strategies necessary for planning for, responding to, and successfully managing change in a complex world.

Chapter 14 by Keith Hunter presents an exercise that explores the factors that drive the emergence of social networks as well as the extraction of value from those networks. The underlying objective of the simulation is to enhance participant understanding of social capital, exchange, and power in social networks. The exercise is built around a simple game using standard playing cards in which participants examine rather complex and influential processes that are associated with the accumulation, adoption, and sustaining of organizational change. As Hunter underscores, much like in a real organization, a group of participants is composed of individuals who operate under a need to maximize their local interests; with their capacity to do so highly dependent on exchange relationships with at least some others who have their own local priorities. By engaging in multiple dyadic interactions with the aim of maximizing the value of the resources (cards) they hold, participants experience the need to negotiate with their counterparts to obtain resources (cards) that will improve their respective situations.

Therese Yaeger concludes Part IV with Chapter 15, focused on generational conflict as participants experience how workers from different age cohorts present themselves as the "best person" for a new leadership role. The basic objective is to gain appreciation of perspectives and challenges of four different generations in today's workforce. Through an experiential role play, participants uncover greater insight into the values and needs of different age groups and how those differences can impact decisions. As Yaeger concludes, through the role play exchanges an underlying hope is that participants will also realize their own shortcomings and biases when working with other age groups. 


\section{The Experiential Exercise End Game: End-Point Engagement}

The final part of the volume, Part V, provides guidance for debriefing experiential exercises. We believe that the debrief is the most crucial part of the learning process associated with using experiential exercises, which unfortunately is often cut short due to time constraints. Deconstructing an exercise in real time, directly following the participants' experience while it is fresh in their minds, is obviously the preferred approach. A challenge with experiential exercises, however, is that they can be very seductive, engaging participants (and facilitators) at such a level that time seems to slip by, leaving little time for true exploration and reflection of the experience. An all too common refrain - "That was fun" - often seems to surpass what was (or was not) learned. Thus, while what might be thought of as immediate teachable moments might be preferable, planned teachable moments still provide powerful learning experiences: in essence, starting the next class or training session with a look back at what transpired in the previous exercise, ensuring sufficient time and attention to the underlying takeaways and lessons from the experience.

In Chapter 16, Gary Wagenheim provides a general framework for thinking about "the debrief," the process by which students are encouraged to critically analyze their individual experience, with the goal of gaining further insights into their learning. Although each of the exercise presentations in the earlier chapters include debriefing guidelines for that particular activity, Wagenheim extends these recommendations though an explicit set of guidelines based on objective, reflective, interpretive, and decisional questions that guide participants in their own personal self-exploration. As he suggests, these four sets of questions are not intended to be as linear as they might initially suggest. In practice, students tend to drift between questions, get stuck on issues, get distracted or run out of energy, and think in ways that may run counter to the sequence. As he argues, given that this type of dialogue is circular - often "messy and sticky, and occasionally dull" - as the facilitator it is important to prompt students to dig deeper with those questions that resonate with them, probing for more information and insight and even simply moving on to other questions if there are no responses. The goal is to prompt student ownership of their learning, helping to promote the ever-important and desired life-long reflective learner.

Chapter 17 by Anthony Buono explores the idea of a Three-Part Journal as a way of enhancing concept application and reflective learning. The chapter presents a structured journaling technique originally created by our Bentley University colleague Edward Zlotkowski as a way of enhancing student experience and development in service-learning engagements. 
Certainly relevant to organizational change, this activity captures: (1) participant thoughts about the experience (written in regular font); (2) how that experience relates to course (or training session) concepts and frameworks (bold face font); and (3) focused reflection (italics), pushing participants to delve into their own feelings and personal aspects of the learning process, from testing assumptions and beliefs, to contemplating new attitudes and behaviors as part of their own growth and development.

The volume concludes in Chapter 18 with some brief reflections by the editors, delving into the value of learning about change through personal experience. Focusing on the integrative development and place of using games, exercises, and simulations in order to better engage with organizational change, the concluding discussion counterbalances the toolkit approach. In this way, the book is a resource for understanding change through experience.

\section{ACKNOWLEDGMENTS}

As noted in the first section of this introductory chapter, this book was inspired by more than a decade of successful Professional Development Workshops (PDWs) at the annual meeting of the Academy of Management (AoM). Two key AoM divisions - the Organization Development and Change Division and the Management Consulting Division - were continually and highly supportive of our efforts, sponsoring the PDWs year after year. We would also like to thank the presenters as well as the participants in these sessions. Our presenters (many of whom are contributors to the volume) were fully open in their willingness to share their materials, engage with the audience, and in general, inform and have fun along the way. Similarly, this book would never have happened without the good-natured participation and involvement by those who attended the workshops, in many instances multiple times over the years. Their reaction to the exercises and subsequent discussions and feedback prompted us to capture the experience in this volume.

The PDW was initially created by Gavin Schwarz with Ian Palmer and Richard Dunford, who played central roles in developing the underlying idea and plan for the workshops. As Ian and Richard gravitated toward academic administration roles, Anthony Buono and Susan Adams became co-developers, working with Gavin to continue the insightful work of our Australian colleagues. Co-writing and editing this volume has been one of the more enjoyable writing projects that we have experienced. It is our hope that the book - and the PDWs that inspired it - will help to stimulate a revival of experiential learning as an important pedagogical approach, 
helping readers and users to more fully understand and appreciate the complexities, nuances, and possibilities inherent in the change process. And still have some fun along the way.

\section{REFERENCES}

Abrahamson, E. (2000). Change without pain. Harvard Business Review, 78(4), $75-79$.

Barbe, W.B., Swassing, R.H., and Milone, M.N. (1979). Teaching through Modality Strengths: Concepts Practices. Columbus, $\mathrm{OH}$ : Zaner-Bloser.

Beer, M. (2006). High Commitment High Performance: How to Build a Resilient Organization for Sustained Advantage. San Francisco, CA: Jossey-Bass.

Boyatzis, R.E. and Kolb, D.A. (1995). From learning styles to learning skills: the executive skills profile. Journal of Managerial Psychology, 10(5), 3-17.

de Caluwé, L. and Vermaak, H. (2002). Learning to Change: A Guide for Organization Change Agents. London: SAGE Publications.

Jamieson, D. and Armstrong, T. (2010). Consulting for change: creating value through client-consultant engagement. In A.F. Buono and D.W. Jamieson (eds), Consultation for Organization Change (pp. 3-13). Charlotte, NC: Information Age Publishing.

Jamieson, D.W., Barnett, R.C., and Buono, A.F. (eds) (2016). Consultation for Organizational Change Revisited. Charlotte, NC: Information Age Publishing.

Johnson, S. (1988). Who Moved My Cheese? An Amazing Way to Deal with Change in your Work and in your Life. New York, NY: G.P. Putnam's Sons.

Kerber, K.W. and Buono, A.F. (2005). Rethinking organizational change: reframing the challenge of change management. Organization Development Journal, 23(3), 23-38.

Kerber, K.W. and Buono, A.F. (2018). The rhythm of change leadership. Organization Development Journal, (Fall), 1-18.

King, S.B. and Wright, M. (2007). Building internal change management capability at Constellation Energy. Organization Development Journal, 25(2), 57-62.

Kolb, D.A. (2014). Experiential Learning: Experience as the Source of Learning and Development. Hoboken, NJ: Pearson FT Press.

Kotter, J. (1996). Leading Change. Cambridge, MA: Harvard Business School Press.

Kotter, J.P. and Cohen, D.S. (2002). The Heart of Change: Real-Life Stories of how People Change their Organizations. Boston, MA: Harvard Business School Press.

Kotter, J.P. and Rathgeber, H. (2016). Our Iceberg is Melting: Changing and Succeeding under any Conditions. New York: Penguin Random House.

Lewin, K. (1945). The research centre for group dynamics at Massachusetts Institute of Technology. Sociometry, 8, 126-35.

Pfeiffer, J.W. and Jones, J.E. (1972). The 1972 Annual Handbook for Group Facilitators. Iowa City, IA: University Associates Press. 